\title{
A Heuristic Approach to Control the Ecovat System
}

\author{
Gijs J. H. de Goeijen, Johann L. Hurink, Gerard J. M. Smit \\ University of Twente, Department of EEMCS \\ Enschede, The Netherlands \\ Email: \{g.j.h.goeijen, j.l.hurink, g.j.m.smit\}@utwente.nl
}

\begin{abstract}
The Ecovat system is a seasonal thermal storage technology developed to supply the heat demand of a neighbourhood of houses throughout the entire year. It consists of a large subterranean water tank which is divided into a number of virtual segments which can be charged or discharged independently from each other. In this work, we present a fast heuristic approach to model and control the charging (store heat) and discharging (withdraw heat) of the Ecovat system. We compare the results obtained with this approach to results obtained with a previously developed integer linear programming model (ILP) of the Ecovat system, which is too slow to be used in practice. The heuristic is found to reduce the computational time from 12+ hours for the ILP model to one second and is thereby suitable to be used in practice. The price for this reduction is an average decrease in performance of $5.2 \%$ and a maximum observed decrease of $12.5 \%$.
\end{abstract}

\section{INTRODUCTION}

In recent years the share of renewable energy sources, such as sun and wind, has increased in an effort to reduce greenhouse gas emissions as well as to reduce the dependence on fossil fuels to supply our energy demands. However, these renewables lead to a new challenge: generation peaks do not always coincide with peaks in the energy demand. To overcome this challenge much research has been done on solutions, such as demand side management (DSM) and energy storage, which both allow for a better matching of supply and demand. In this work we focus on a specific thermal energy storage solution.

Thermal storage technologies can be divided into three categories. The first is sensible heat storage, in which energy is stored by increasing the temperature of the storage medium, usually water or rock. Examples of sensible heat storage are water tanks [1], [2], aquifers [3] and rock beds [4]. The second is latent heat storage, in which energy is stored due to a phase change in the storage medium, called phase change materials (PCM). Compared to sensible heat storage latent heat storage achieves higher energy densities, but at higher investment costs. The use of PCM for thermal energy storage is reviewed in [5], [6]. The final category is chemical storage, in which energy is stored by means of reversible chemical reactions. Compared to the previous two categories chemical storage achieves even higher energy densities, but in general is also more expensive. A review of chemical storage technologies is presented in [7].

In this paper we consider the Ecovat system [8]. The system consists of the Ecovat buffer, which is a large underground water tank, accompanied by a number of devices to charge and discharge the buffer; PVT panels, heat pumps and a resistance heater. The Ecovat buffer is a sensible heat storage technology, i.e. charging of the buffer causes the water to increase in

978-1-5386-4505-5/18/\$31.00 2018 IEEE temperature. The feature that differentiates the Ecovat buffer from other water tanks is the fact that water is not pumped into or out of the buffer when charging or discharging. Instead, the charging and discharging of the buffer is done by heat exchangers integrated into the buffer walls. The Ecovat buffer is divided into a number of horizontal segments, which can be charged or discharged individually. However, these segments are not physically separated from each other. The charging and discharging of the buffer is done in such a way that the stratification inside the buffer remains intact, as this has been shown to increase efficiency [9], [10].

In previous work we modelled the Ecovat system and its control by means of an integer linear programming (ILP) model [11]. Based on predictions for the heat demand, ambient temperature and energy prices during a given period the ILP model determines a charging and discharging strategy for the Ecovat system for that period. However, due to the complexity of the problem, the ILP model can only be solved for short time periods (up to a few days). To be able to apply the model to longer time periods (preferably one year), a rolling horizon approach is employed in the model. This approach optimizes two days in advance with the ILP model, while only the first of those days is realised. The results in [11] show that a weakness of this model is that it might lead to a situation where the model is not able to sufficiently incorporate seasonal effects.

In follow up work [12], we extended the ILP model with a long-term planning, which determines, based on historical heat demand data and predicted energy prices, a target state of charge for the buffer for every day within the optimization horizon. The ILP model is then penalized whenever the state of charge drops below this target during the optimization. This extension allowed the model to better incorporate seasonal effects as shown in [12]. Furthermore, it was shown that the model is very robust against prediction errors.

While the ILP model gives good solutions it is computationally expensive. For example, a rolling horizon optimization spanning a complete year takes 12 hours or more. This means that on average a time of almost two minutes (and in some extreme cases a much longer time) is required to determine a strategy for a given day. For integration into a neighbourhood level DSM system these computation times are too long. Even more importantly, when controlling a real Ecovat system a method capable of making fast (sub-second) decisions will be necessary. In this paper we present such a fast, heuristic control approach for the Ecovat system. The goal of this heuristic is to provide fast solutions while retaining an acceptable level of accuracy when compared to solutions given by the ILP model.

The remainder of this paper is structured as follows: in 


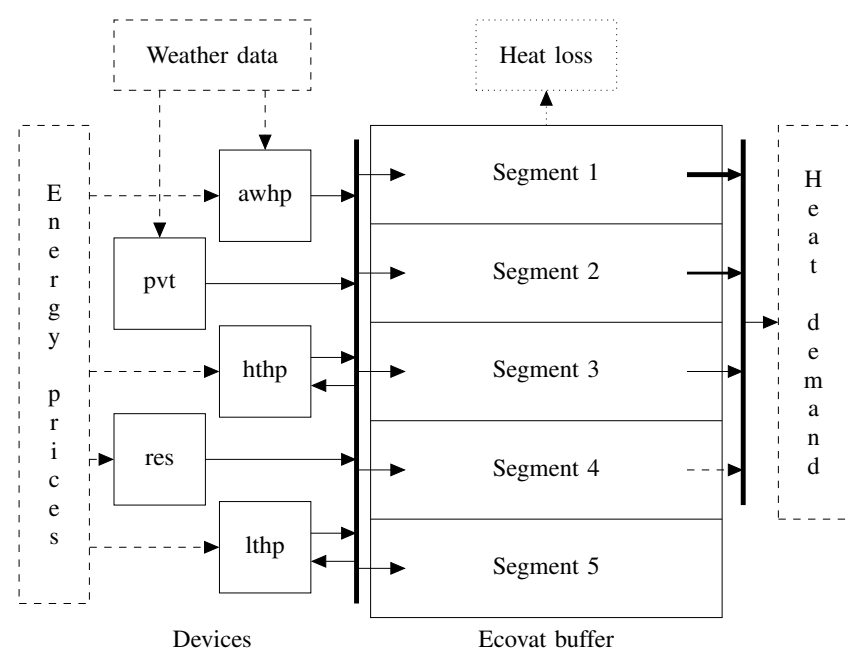

Fig. 1. Schematic overview of the Ecovat system, consisting of the Ecovat buffer and a number of devices to charge the buffer, namely PVT panels (pvt), a resistance heater (res) and heat pumps (lthp, hthp and awhp).

Section II the developed heuristic approach is presented, while in Section III the input data is described. Next, in Section IV the results of the comparison between the heuristic and the ILP model are presented and discussed. Finally, in Section V conclusions are presented and future work is outlined.

\section{HeURISTIC APPROACH}

The goal of the Ecovat system is to supply the heat demand of a neighbourhood of houses for both space heating and tap water, while minimizing the cost for supplying this energy. To ensure that enough energy is available in the buffer to supply this demand, the buffer is charged using photovoltaic-thermal (PVT) panels, heat pumps and a resistance heater by using locally generated energy or energy bought on the energy market, preferably when the price is low or even negative.

In Fig. 1 a schematic overview of the Ecovat system is shown. The Ecovat buffer is divided into five segments, each with a different temperature. To avoid stratification issues the temperature within the segments needs to be decreasing, with Segment 1 having the highest temperature. In the left part of Fig. 1 the devices to charge the buffer are shown. This charging (and discharging) is done through heat exchangers integrated into the buffer walls. The PVT panels (pvt in Fig. 1) can charge the buffer if their output temperature is high enough. The output of these panels can only be connected to the bottom segment, since in general the temperature is relatively low. At the same time the bottom segment will be kept at a low temperature so that the PVT panels can be cooled to increase their efficiency. The resistance heater (res in Fig. 1) is able to add a large amount of energy to the buffer, however, it does so at a low efficiency. This makes it an ideal device to charge the buffer during intervals with negative energy prices.

The remaining devices are a number of heat pumps, namely an air-water heat pump (awhp in Fig. 1) that can charge the buffer using the outside air as heat source and two water-water heat pumps that use one of the buffer segments as a heat source and another segment (with higher temperature) as heat sink.
Because of the large temperature range in the buffer (from about $5{ }^{\circ} \mathrm{C}$ in the bottom segment up to $90{ }^{\circ} \mathrm{C}$ in the top segment) one of the water-water heat pumps is used to cover the low temperatures and one to cover the high temperatures (lthp and hthp respectively in Fig. 1). The exact temperature ranges of the heat pumps are set as parameters in the heuristic.

The heuristic approach developed in this work is based on the ILP model developed in [11] and [12]. The input data for both methods consists of energy prices, weather data and heat demand for every interval in the time horizon. We define the set of intervals in the time horizon as $\mathcal{I}=\left\{1,2, \ldots, N_{i n t}\right\}$, with $N_{\text {int }}$ the number of intervals. As common for DSM, we take the length of an interval to be 15 minutes. We define the set of segments in the Ecovat buffer as $\mathcal{S}=\left\{1,2, \ldots, N_{\text {seg }}\right\}$, with $N_{\text {seg }}=5$ the number of segments. The input data consists of a vector containing the energy prices $\mathbf{p}=\left(p_{1}, p_{2}, \ldots, p_{N_{i n t}}\right)$, a vector containing the ambient temperature $\mathbf{T}^{a}=\left(T_{1}^{a}, T_{2}^{a}, \ldots, T_{N_{\text {int }}}^{a}\right)$, a vector containing the global radiation $\mathbf{G}=\left(G_{1}, G_{2}, \ldots, G_{N_{i n t}}\right)$ and a vector containing the heat demand $\mathbf{d}=\left(d_{1}, d_{2}, \ldots, d_{N_{i n t}}\right)$ for every interval in the time horizon. In simulations these values may be based on historical data and predictions, while in real operation real time measurements may be used to update these values. The goal of the heuristic is to determine for each time interval the use of the devices while minimizing costs, and thereby identical to that of the ILP model.

Similar to the ILP model, the heuristic starts by generating a target, $\mathbf{V}=\left(V_{1}, V_{2}, \ldots, V_{\left\lfloor N_{\text {int }} / 96\right\rfloor}\right)$, for the useful energy in the buffer at the end of every day [12]. Here useful energy is defined as energy that can be used to supply the heat demand, i.e. energy at temperatures higher than the given demand temperature $T_{d}$ :

$$
U_{i}=\sum_{s \in \mathcal{S}} m_{s} c_{p} \max \left\{T_{i, s}-T_{d}, 0\right\}
$$

where $U_{i}$ is the useful energy at interval $i, T_{i, s}$ is the temperature of segment $s$ during interval $i, m_{s}$ is the mass of segment $s$ and $c_{p}$ is the specific heat coefficient of water. The purpose of $\mathbf{V}$ is to make sure the buffer has enough energy stored at times of high demand, in general during winter.

To describe the workings of the heuristic approach we will first give an outline of the heuristic and subsequently elaborate on the different steps of the heuristic in the rest of this section. During every interval $i$ in the time horizon the following steps are performed:

1) Determine the maximum accepted energy price for the devices to run.

2) Calculate the output temperature of the PVT panels.

3) Decide whether the PVT panels, lthp and/or hthp run.

4) Decide which segment supplies the heat demand and whether the resistance heater and awhp run.

5) Calculate the heat losses in the Ecovat during interval $i$.

6) Calculate the energy change due to the decisions made.

7) Update temperatures $T_{i, s}$ of the segments.

8) Calculate the costs incurred due to the decisions made.

After the last interval in the time horizon the total costs incurred over the entire horizon are calculated. The objective of both the ILP model and the heuristic is to minimize this total cost. 
For time interval $i$ the heuristic considers only the current state of the buffer, i.e. the temperatures of the buffer segments, as well the input data for that interval. The heuristic does not take any future data into account, except indirectly through the targets $\mathbf{V}$. The heuristic uses a set of 'rules of thumb' to determine, based on the current state and input data, the devices that will be connected to specific buffer segments. Whether a device is connected to a buffer segment is modelled by the binary variable $x_{i, s}^{d e v}$, where dev is the device in question ( $p v t, r e s, a w h p, l t h p, h t h p$ and dem, whereby dem is the heat demand). If $x_{i, s}^{d e v}=1$ device dev is connected to segment $s$ during interval $i$ and if $x_{i, s}^{d e v}=0$ it is not connected. An important constraint of the Ecovat system is that only one of these devices can be connected to each buffer segment during any time interval. In the following the steps taken by the heuristic are explained in more detail.

Step 1: Based on the target $V_{d}$ for a given day $d$ and the useful energy content $U_{\lceil i / 96\rceil-1}$ of the buffer at the start of that day, the maximum accepted energy price $\bar{p}_{d}$ is determined. The value of $p_{d}$ is used to decide whether devices are turned on during interval $i$. Here three cases are distinguished: $(i)$ the useful energy content of the buffer is below target, (ii) the useful energy content of the buffer is above target, and (iii) the buffer is almost full. More precisely, $\bar{p}_{d}$, is given by:

$$
\bar{p}_{d}=\left\{\begin{array}{ll}
0.01 \cdot\left(\bar{U}-15000-U_{\lceil i / 96\rceil-1}\right) & \text { if } U_{\lceil i / 96\rceil-1}> \\
0 & \bar{U}-15000 \\
241 \cdot\left(1-\frac{U_{\lceil i / 96\rceil-1}}{V_{d}}\right)^{2}+9 & \text { if } U_{\lceil i / 96\rceil-1} \geqslant V_{d} \\
& \text { if } U_{\lceil i / 96\rceil-1}<V_{d}
\end{array},\right.
$$

where $\bar{U}$ is the maximum amount of useful energy that can be stored in the buffer. The value of $\bar{U}$ depends on the maximum allowed temperatures $\bar{T}_{s}$ in the buffer. $\bar{U}$ can be calculated using equation (1) by substituting $T_{i, s}$ with $\bar{T}_{s}$. The maximum accepted energy price ranges from 9 to $250 € / \mathrm{MWh}$ when the useful energy content of the buffer drops below the target and from 0 to $-150 € / \mathrm{MWh}$ when the useful energy content of the buffer is approaching $\bar{U}$. The constants used have experimentally been found to work well, but can easily be changed if for example the energy prices are expected to change in the future.

Step 2: The output temperature $T_{i}^{\text {out }}$ of the PVT panels at interval $i$ is calculated as in [11]:

$$
T_{i}^{o u t}=\frac{2 \dot{m} c_{p} T_{i}^{i n}-a_{t h} A T_{i}^{i n}+2 A \eta_{0}^{t h} G_{i}+2 a_{t h} A T_{i}^{a}}{a_{t h} A+2 \dot{m} c_{p}},
$$

where $\dot{m}$ is the mass flow rate of the liquid through the PVT panels, $T_{i}^{i n}$ is the input temperature of that liquid, $a_{t h}$ is the thermal loss coefficient of the PVT panels, $A$ is the surface area of the PVT panels, $G_{i}$ is the global radiation, $T_{i}^{a}$ is the ambient temperature and $\eta_{0}^{\text {th }}$ is the thermal efficiency of the PVT panels at a reduced temperature of zero. Using this output temperature the efficiency of the PVT panels can be calculated, which is required in step 6 of the heuristic. For this we first need the reduced temperature $T_{i}^{r}$, which is given by:

$$
T_{i}^{r}= \begin{cases}\frac{\frac{T_{i}^{\text {in }}+T_{i}^{\text {out }}}{2}-T_{i}^{a}}{G_{i}} & \text { if } G_{i}>0 \\ 0 & \text { otherwise. }\end{cases}
$$

Finally the thermal and electrical efficiencies, $\eta_{i}^{t h}$ and $\eta_{i}^{e l}$ respectively, of the PVT panels are modelled as a linear function of $T_{i}^{r}$ [13], [14]:

$$
\begin{gathered}
\eta_{i}^{t h}=\eta_{0}^{t h}-a_{t h} T_{i}^{r} \\
\eta_{i}^{e l}=\eta_{0}^{e l}-a_{e l} T_{i}^{r},
\end{gathered}
$$

where $a_{e l}$ is the electrical loss coefficient of the PVT panels and $\eta_{0}^{e l}$ is the electrical efficiency of the PVT panels at a reduced temperature of zero. For a more extensive discussion on the modelling of the PVT panels we refer to the description of the ILP model presented in [11].

Step 3: Since the temperature of the bottom segment of the buffer has to stay below $5{ }^{\circ} \mathrm{C}$ to cool the PVT panels the lthp is forced to run to transfer heat from the bottom segment to other segments if this condition is not fulfilled. In a similar way, based on the technical specifications of the heat pumps and temperature bounds $\bar{T}_{s}$ on the buffer segments, the hthp is forced to run if similar conditions are not fulfilled in the given interval. Finally, the PVT panels are connected to the bottom segment of the buffer only if the output temperature of the PVT panels is higher than the temperature in the bottom segment and the lthp is not connected to that segment. Furthermore, the lthp and hthp must always be connected to two segments if they are turned on, a heat source and a heat sink, labeled $l t h p_{c}$ and $l t h p_{w}$ respectively for the low temperature heat pump and $h t h p_{c}$ and $h t h p_{w}$ for the high temperature heat pump. This leads to the following constraints for running the lthp and hthp:

$$
\begin{array}{rlrl}
\sum_{s \in \mathcal{S}} x_{i, s}^{l t h p_{w}} & =\sum_{s \in \mathcal{S}} x_{i, s}^{l t h p_{c}} & \forall i \in \mathcal{I} \\
\sum_{s \in \mathcal{S}} x_{i, s}^{h t h p_{w}}=\sum_{s \in \mathcal{S}} x_{i, s}^{h t h p_{c}} & \forall i \in \mathcal{I} .
\end{array}
$$

Step 4: Once it has been determined for interval $i$ how the lthp, hthp and the PVT panels are used, the demand, resistance heater and awhp are considered. Due to the restriction that only one device can be connected to a segment during a time interval the choices for these devices strongly influence each other. Furthermore, as the heat demand has to be fulfilled at all times one of the segments has to be assigned to supplying the heat demand during interval $i$. The rule of thumb here is that the demand is assigned to a segment that minimally constrains the ability of the resistance heater and awhp to run and the resistance heater is prioritized over the awhp, because of the larger profit it can generate during intervals with negative energy prices. More precisely, the heuristic will try to use the resistance heater if the energy price of the current time interval is equal to or lower than the maximum accepted energy price $\bar{p}_{d}$. Similarly, the heuristic will try to use the awhp if the current energy price is equal to or lower than $\bar{p}_{d}$ multiplied with the coefficient of performance of the awhp. However, due to the temperature bounds $\bar{T}_{s}$ on the buffer segments and the 
technical specifications of the awhp this may not always be possible even if the price is low enough.

Step 5: In this step the heat losses to the environment in interval $i$ are taken into account. For this we use the same approach as in the ILP model [11], i.e. we assume the relative heat loss $Q_{i, s}^{\text {loss }}$ during interval $i$ in segment $s$ is given by:

$$
Q_{i, s}^{l o s s}=\left(1-(1-\beta)^{\frac{1}{4380}}\right)\left(T_{i, s}-T_{e}\right) m_{s} c_{p},
$$

where $\beta$ is the heat loss factor of the buffer over six months, $T_{e}$ is the temperature of the environment and the factor 4380 is the number of hours in six months. The heat loss factor of an Ecovat buffer over six months has been calculated in an internal study of the company. Ideally, measurements from a real Ecovat system should be used instead. However, at the time of writing these were not available yet.

Step 6: The amount of energy added to or extracted from the buffer depends on the capacities of the devices, $C^{d e v}$, and their coefficients of performance, $C O P^{d e v}$. The (thermal) energy changes, $Q_{i, s}^{d e v}$ due to the different devices are given by:

$$
\begin{aligned}
& Q_{i, s}^{p v t}= \begin{cases}0 & \text { if } s=1, \ldots, 4 \\
\eta_{i}^{t h} G_{i} A x_{i, s}^{p v t} & \text { if } s=5\end{cases} \\
& Q_{i, s}^{r e s}=C^{r e s} x_{i, s}^{r e s} \\
& Q_{i, s}^{a w h p}=C^{a w h p} C O P^{a w h p} x_{i, s}^{a w h p} \\
& Q_{i, s}^{l t h p_{w}}=C^{l t h p} C O P^{l t h p} x_{i, s}^{l t h p_{w}} \\
& Q_{i, s}^{l t h p_{c}}=-C^{l t h p}\left(C O P^{l t h p}-1\right) x_{i, s}^{l t h p_{c}} \\
& Q_{i, s}^{h t h p_{w}}=C^{h t h p} C O P^{h t h p} x_{i, s}^{h t h p_{w}} \\
& Q_{i, s}^{h t h p_{c}}=-C^{h t h p}\left(C O P^{h t h p}-1\right) x_{i, s}^{h t h p_{c}} \\
& Q_{i, s}^{d e m}=-d_{i} x_{i, s}^{d e m} \text {. }
\end{aligned}
$$

Note that even though $C^{\text {dev }} C O P^{d e v}$ thermal energy is transferred to the heat sink (equations (13) and (15)) only $C^{\text {dev }}\left(C O P^{d e v}-1\right)$ comes from the heat source (equations (14) and (16)). The remainder comes from the electrical energy used to operate the heat pump. For simplicity we assume no heat losses in the heat pump operation. The electrical energy generated by the PVT panels is given by:

$$
E_{i}^{p v t}=\sum_{s \in \mathcal{S}} \eta_{i}^{e l} G_{i} A x_{i, s}^{p v t} .
$$

Step 7: The temperature change in the buffer segments due to the energy changes during an interval are calculated by:

$$
\begin{aligned}
T_{i, s} & =T_{i-1, s}+\frac{\Delta t}{m_{s} c_{p}}\left(Q_{i, s}^{p v t}+Q_{i, s}^{r e s}+Q_{i, s}^{a w h p}+Q_{i, s}^{l t h p_{w}}\right. \\
& \left.+Q_{i, s}^{l t h p_{c}}+Q_{i, s}^{h t h p_{w}}+Q_{i, s}^{h t h p_{c}}+Q_{i, s}^{\text {dem }}+Q_{i, s}^{l o s s}\right),
\end{aligned}
$$

where $\Delta t$ is the interval length.

Step 8: The total energy costs incurred during interval $i, K_{i}$, is calculated by:

$$
\begin{aligned}
K_{i}=\Delta t & p_{i} \sum_{s \in \mathcal{S}}\left(C^{r e s} x_{i, s}^{r e s}+C^{a w h p} x_{i, s}^{a w h p}\right. \\
& \left.+C^{l t h p} x_{i, s}^{l t h p_{c}}+C^{h t h p} x_{i, s}^{h t h p_{c}}\right)-E_{i}^{p v t} .
\end{aligned}
$$

Due to equation (7) it does not matter whether we use $x_{i, s}^{l t h p_{c}}$ or $x_{i, s}^{l t h p_{w}}$ to determine if the lthp is running during interval $i$. The same holds for the hthp. At the end of the overall simulation the total cost over the time horizon can be calculated by adding up the costs per interval:

$$
K_{t o t}=\sum_{i \in \mathcal{I}} K_{i}
$$

The described heuristic solves the same problem as the ILP model. However, the difference is that the ILP model solves the problem for a set of time intervals simultaneously while the heuristic solves the problem iteratively for successive time intervals. Due to this difference some simplifying assumptions have to be made compared to the ILP (the rules of thumb discussed in this section), which leads to the heuristic having a decreased performance when compared to the ILP model.

\section{Simulation SETUP}

To determine the performance of the heuristic we compare the obtained results with the results from the ILP model in [11] and [12]. As such we use the same input data as used in those papers. This means that the used heat demand profiles and ambient temperatures are averages taken over the period 20052011. The real energy prices from the Dutch imbalance market from the years 2011, 2013, 2014 and 2015 have been used (the year 2012 was excluded to avoid normalization issues because it is a leap year). The remaining input parameters are the same as those used in [11] (summarized in Table I). Constant COP values are used to be able to compare the results of the heuristic approach to the ILP model from [11].

For the initial generation of the targets, $\mathbf{V}$, the same procedure as in [12] is used. More specifically, an optimization problem is solved that determines a charging strategy to supply the heat demand throughout the year at minimum cost. For this problem only the historical data for the heat demand and energy prices are used as input. Even though this optimization problem is a great oversimplification of the actual Ecovat system it gives a good indication for the desired useful energy content of the buffer throughout the year. For example, it makes sure there is enough energy in the buffer to cover all heat demand during winter. As in [12], two cases are considered. In the first case perfect predictions of the energy prices are available for the

TABLE I

INPUT PARAMETERS FOR THE HEURISTIC MODELLING THE ECOVAT SYSTEM.

\begin{tabular}{c|c|c|c}
\hline Parameter & Value & Parameter & Value \\
\hline$N_{\text {int }}$ & 35040 & $\eta_{0}^{t h}$ & 0.73 \\
$\Delta t$ & $900 \mathrm{~s}$ & $a_{t h}$ & 7.25 \\
$N_{\text {seg }}$ & 5 & $\eta_{0}^{e l}$ & 0.1 \\
$m_{s}$ & $\left(1.04 \cdot 10^{6}, 1.04 \cdot 10^{6}\right.$, & $a_{e l}$ & 0.44 \\
& $1.04 \cdot 10^{6}, 9.11 \cdot 10^{5}$, & $\beta$ & 0.08 \\
& $\left.9.11 \cdot 10^{5}\right) \mathrm{kg}$ & $C^{a w h p}$ & $9 \mathrm{~kW}$ \\
$c_{p}$ & $4.186 \frac{\mathrm{kJ}}{\mathrm{kg}{ }^{\circ} \mathrm{C}}$ & $C^{l t h p}$ & $15 \mathrm{~kW}$ \\
$T_{0}, s$ & $(90,75,50,30,5){ }^{\circ} \mathrm{C}$ & $C^{h t h p}$ & $15 \mathrm{~kW}$ \\
$\bar{T}_{s}$ & $(90,90,78,48,5){ }^{\circ} \mathrm{C}$ & $C^{\text {res }}$ & $1000 \mathrm{~kW}$ \\
$T_{d}$ & $40 \mathrm{or} 60{ }^{\circ} \mathrm{C}$ & $C O P^{a w h p}$ & 2.686 \\
$T_{e}$ & $15{ }^{\circ} \mathrm{C}$ & $C O P^{l t h p}$ & 2.851 \\
$\dot{m}$ & $0.018 \frac{\mathrm{kg}}{\mathrm{s}}$ & $C O P^{h t h p}$ & 3.681 \\
\hline \multicolumn{2}{|c}{}
\end{tabular}


TABLE II

COMPARISON BETWEEN THE ILP MODEL AND HEURISTIC.

\begin{tabular}{l|cc|cc|c}
\hline & \multicolumn{2}{|c|}{$\begin{array}{c}\text { ILP } \\
\text { Useful energy }(\mathrm{kWh})\end{array}$} & $\begin{array}{c}\text { Costs }(€) \\
\text { Ceuristic } \\
\text { Useful energy }(\mathrm{kWh})\end{array}$ & $\begin{array}{c}\text { Difference in costs } \\
\%\end{array}$ \\
\hline$T_{d}=40^{\circ} \mathrm{C}, 2011, \mathrm{NP}$ & -22091 & 148014 & -21406 & 141615 & 3.1 \\
$T_{d}=40^{\circ} \mathrm{C}, 2011, \mathrm{PP}$ & -22698 & 115764 & -22073 & 115837 & 2.8 \\
$T_{d}=40^{\circ} \mathrm{C}, 2013, \mathrm{NP}$ & -41607 & 117121 & -40847 & 113889 & 1.8 \\
$T_{d}=40^{\circ} \mathrm{C}, 2013, \mathrm{PP}$ & -41703 & 114719 & -40976 & 111102 & 1.7 \\
$T_{d}=40^{\circ} \mathrm{C}, 2014, \mathrm{NP}$ & -27773 & 110122 & -27158 & 105850 & 2.2 \\
$T_{d}=40^{\circ} \mathrm{C}, 2014, \mathrm{PP}$ & -27865 & 110383 & -27445 & 103143 & 1.5 \\
$T_{d}=40^{\circ} \mathrm{C}, 2015, \mathrm{NP}$ & -47077 & 131081 & -48089 & 121663 & -2.1 \\
$T_{d}=40^{\circ} \mathrm{C}, 2015, \mathrm{PP}$ & -47077 & 131081 & -47931 & 125347 & 1.8 \\
\hline$T_{d}=60^{\circ} \mathrm{C}, 2011, \mathrm{NP}$ & -21008 & 78958 & -18379 & 77896 & 10.7 \\
$T_{d}=60^{\circ} \mathrm{C}, 2011, \mathrm{PP}$ & -22351 & 54101 & -19950 & 64508 & 4.8 \\
$T_{d}=60^{\circ} \mathrm{C}, 2013, \mathrm{NP}$ & -40709 & 59450 & -38740 & 58350 & 2.9 \\
$T_{d}=60^{\circ} \mathrm{C}, 2013, \mathrm{PP}$ & -41317 & 52539 & -39275 & 51132 & 3.7 \\
$T_{d}=60^{\circ} \mathrm{C}, 2014, \mathrm{NP}$ & -26409 & 57074 & -25804 & 54728 & 10.5 \\
$T_{d}=60^{\circ} \mathrm{C}, 2014, \mathrm{PP}$ & -27166 & 48090 & -26170 & 48377 & 10.9 \\
$T_{d}=60^{\circ} \mathrm{C}, 2015, \mathrm{NP}$ & -51615 & 61856 & -46173 & 60204 & \\
$T_{d}=60^{\circ} \mathrm{C}, 2015, \mathrm{PP}$ & -52225 & 53687 & -46516 & 54252 & \\
\hline
\end{tabular}

entire year (PP case), which means that charging of the buffer in the aforementioned optimization problem is done in intervals with negative energy prices as much as possible. In the second case no predictions for the energy prices are available (NP case). In the NP case the targets, $\mathbf{V}$, are determined by simply distributing the charging equally over the entire year. The only difference between both cases is in the target generation.

In the ILP model from [12] a maximum energy price, $\bar{p}_{d}^{i l p}$, of $9 € / \mathrm{MWh}$ is accepted when the useful energy content, $U_{\lceil i / 96\rceil}$, of the buffer is above the target, $V_{d}$. For the results presented in this paper $\bar{p}_{d}^{i l p}$ has been set to $0 € / \mathrm{MWh}$ when $U_{\lceil i / 96\rceil} \leqslant V_{d}$, the same as $\bar{p}_{d}$ in equation (2), to better compare the results from both approaches.

\section{REsults}

The goal of the presented control heuristic for the Ecovat system is to provide a faster method than the ILP model presented in [11] and [12]. To investigate the relative performance of the two approaches 16 cases have been simulated. These cases were created by all possible combinations of the energy prices from four years, two demand temperatures (40 and 60 ${ }^{\circ} \mathrm{C}$ ) and two sets of target values (PP vs NP case). The results of these simulations are summarized in Table II, where both the costs (with negative costs meaning profit) and the useful energy content of the buffer at the end of the time horizon are given. The last column in Table II gives the relative difference in costs between the two approaches.

We can see that for a demand temperature, $T_{d}$, of $40{ }^{\circ} \mathrm{C}$ the differences between the ILP and heuristic is only a few percent. As an example; in Fig. 2 the temperature evolution of the five segments inside the Ecovat buffer is shown for both the ILP model and the heuristic for the PP case, using energy price data from 2014 and a demand temperature of $40{ }^{\circ} \mathrm{C}$. There are some small differences between the two approaches, but in general both approaches give very similar results.

There are two at first sight unexpected results in Table II. This concerns the two cases using the energy price data from 2015 with a demand temperature of $40{ }^{\circ} \mathrm{C}$. First, note that the PP and NP cases both give exactly the same results for the ILP model. Second, the heuristic in these cases gives better results than the ILP model. The first observation can be explained by the high number of intervals with a negative energy price in 2015. The only difference between the two cases is in the target values calculated at the start of the model. However, due to the high amount of intervals with a negative energy price the useful energy content of the buffer never drops below the target value for both the PP and NP cases meaning these cases become effectively the same.

The second observation can be explained by the fact that the heuristic starts decreasing the maximum energy price it is willing to accept when the buffer is almost full, while the ILP does not. Because of the complexity of the model the ILP employs a rolling horizon, in which the ILP model in each step considers input data for two days at a time. Due to this it is possible for the ILP model to fully charge the buffer even though some intervals with very low energy prices might still come up at a later date, leading to the heuristic outperforming the ILP model. If a constraint is added to the ILP model to start accepting lower maximum energy prices when the buffer is

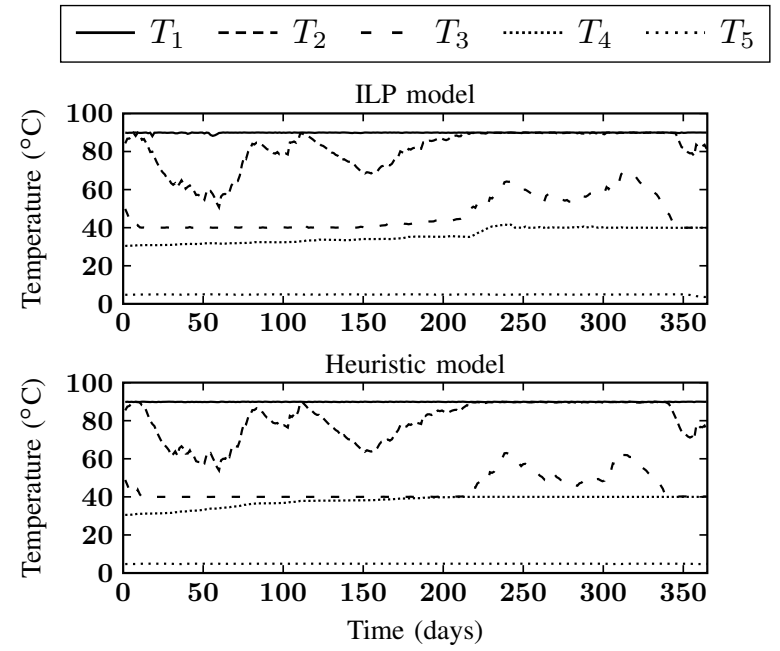

Fig. 2. Temperature evolution inside the Ecovat buffer for the PP case using energy price data from 2014 and a demand temperature $T_{d}=40^{\circ} \mathrm{C}$ 


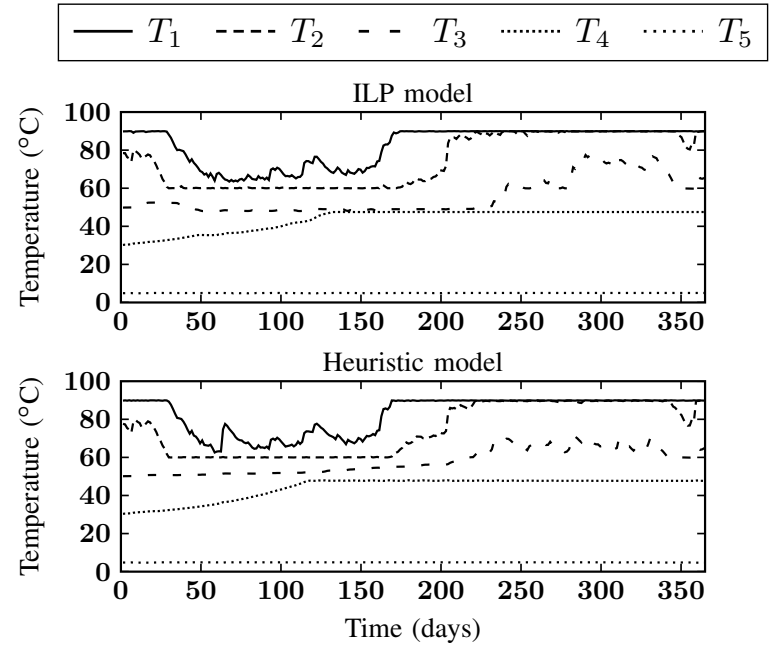

Fig. 3. Temperature evolution inside the Ecovat buffer for the NP case using energy price data from 2011 and a demand temperature $T_{d}=60{ }^{\circ} \mathrm{C}$.

almost full, like the heuristic does, it outperforms the heuristic as expected (costs of -50550 and -50629 are obtained for the NP and PP cases respectively).

Comparing the results of both approaches for a demand temperature of $60{ }^{\circ} \mathrm{C}$ we can see from Table II that the relative difference between the approaches becomes larger, up to $12.5 \%$ for the NP case using energy price data from 2011. Fig. 3 shows the temperature evolution within the buffer for both approaches in this case. As expected, the differences between both approaches are larger than in Fig. 2. However, even in this case the structure of the results looks very similar. The average decrease in performance is $5.2 \%$, excluding the 2015 cases with a demand temperature of $40{ }^{\circ} \mathrm{C}$. In general we expect the decrease in performance to be larger for higher demand temperatures as shown in Table II.

From Table II we observe the same trend when comparing the NP and PP cases as in [12], namely the difference between the two cases is small. In general the PP case does slightly better when considering costs, however, the useful energy content of the buffer at the end of the time horizon is slightly lower.

We observe that the cases in which the relative difference between the approaches is largest are the cases in which the buffer is either almost empty or almost full at some time during the year. Intuitively it makes sense that the advantage of being able to consider future input data, which the ILP model has over the heuristic, is largest in these extreme cases. However, even in these cases the largest observed difference is only $12.5 \%$. Note that the ILP model gives an upper bound on the performance of the heuristic by additionally using input data for future time intervals to determine the optimal strategy for the current interval. Given that in a real world situation it is unlikely to have very accurate predictions for the energy prices, a worst case difference of $12.5 \%$ between both approaches seems to be an acceptable decrease in performance. On the other hand the computational time difference between the approaches is very significant. For most cases the ILP model requires around 12 hours to obtain a solution for a complete year (even up to a few days in some cases) while the heuristic only requires around a second to obtain a solution. This makes the heuristic a suitable method to control the Ecovat within a decentralized energy management setting.

\section{CONCLUSION}

In this work we have presented a heuristic approach for the control of the Ecovat system, with the goal of providing a fast alternative to the ILP model presented in [11] and [12], while retaining an acceptable level of accuracy. The presented heuristic reduces the computational time from $12+$ hours for the ILP model to a second for the heuristic, with at most a $12.5 \%$ decrease in performance. However, on average the decrease in performance is only $5.2 \%$.

In future work we plan to incorporate the heuristic presented here in the DEMKit simulation platform [15], designed to perform hybrid energy DSM simulations. Furthermore, the heuristic can also be used for the control of a real Ecovat system due to the very fast decision making it provides.

\section{ACKNOWLEDGMENT}

The authors thank the Netherlands Enterprise Agency (RVO) for their support through projects TEGB114024 and TES0114004.

\section{REFERENCES}

[1] R. Oliveski, A. Krenzinger, and H. Vielmo, "Comparison between models for the simulation of hot water storage tanks," Solar Energy, vol. 75, p. 121, 2003.

[2] A. Rahman, A. Smith, and F. N., "Performance modeling and parametric study of a stratified water thermal storage tank," Applied Thermal Engineering, vol. 100, p. 668, 2016.

[3] J. Dickinson, N. Buik, M. Matthews, and A. Snijders, "Aquifier thermal energy storage: theoretical and operational analysis," Géotechnique, vol. 59, p. 249, 2009.

[4] M. Hänchen, S. Brückner, and A. Steinfeld, "High-temperature thermal storage using a packed bed of rocks - heat transfer analysis and experimental validation," Applied Thermal Engineering, vol. 31, p. 1798, 2011.

[5] A. Sharma, V. Tyagi, C. Chen, and D. Buddhi, "Review on thermal energy storage with phase change materials and applications," Renewable and Sustainable Energy Reviews, vol. 13, p. 318, 2009.

[6] D. Zhou, C. Zhao, and Y. Tian, "Review on thermal energy storage with phase change materials (pcms) in buildings applications," Applied Energy, vol. 92, p. 593, 2012.

[7] T. Yan, R. Wang, T. Li, L. Wang, and I. Fred, "A review of promising candidate reactions for chemical heat storage," Renewable and Sustainable Energy Reviews, vol. 43, p. 13, 2015.

[8] "Ecovat Renewable Energy Technologies BV." www.ecovat.eu, accessed on March 1, 2017.

[9] Z. Lavan and J. Thompson, "Experimental study of thermally stratified hot water storage tanks," Solar Energy, vol. 19, p. 519, 1977.

[10] M. Rosen, "The exergy of stratified thermal storages," Solar Energy, vol. 71, p. 173, 2001.

[11] G. de Goeijen, G. Smit, and J. Hurink, "An integer linear programming model for an ecovat buffer," Energies, vol. 9, p. 592, 2016.

[12] _ _ "Improving an integer linear programming model of an ecovat buffer by adding long-term planning," Energies, vol. 10, p. 2039, 2017.

[13] H. Zondag, "Flat-plate pv-thermal collectors and systems: A review," Renewable and Sustainable Energy Reviews, vol. 12, p. 891, 2008.

[14] R. Dupeyrat, C. Ménézo, and S. Fortuin, "Study of the thermal and electrical performances of pvt solar hot water system," Energy and Buildings, vol. 68, p. 751, 2014.

[15] G. Hoogsteen, "A cyber-physical systems perspective on decentralized energy management," Ph.D. dissertation, University of Twente, PO Box 217, 7500 AE Enschede, The Netherlands, dec 2017. 